\title{
Predicting data quality of proxy reports in egocentric network studies
}

\author{
Tobias H. Stark $^{\mathrm{a}, *}$, Volker Stocké ${ }^{\mathrm{b}}$ \\ ${ }^{\text {a }}$ ERCOMER (European Research Centre on Migration and Ethnic Relations), Utrecht University/ICS, Padualaan 14, 3584 CH, Utrecht, The Netherlands \\ ${ }^{\mathrm{b}}$ Department of Social Sciences, University of Kassel, Kassel, Germany
}

\section{A R T I C L E I N F O}

Volker Stocké passed way in 2017 after we designed this study. This publication is dedicated to his memory

\section{Keywords:}

Egocentric network study

Proxy reporting

Congruence

Name interpreter question

Survey satisficing

\begin{abstract}
A B S T R A C T
Egocentric network studies and many general population surveys rely on proxy reports about network contacts of study participants that are asked in name interpreter questions. A central concern is the extent to which proxy reports match the answers these contacts would give themselves if they would be directly interviewed. Based on the theory of survey satisficing, the present research proposes a theoretical framework that allows predicting when proxy reports are likely to match self-reports. Congruence is higher if respondents possess the motivation and ability to answer a proxy question effortfully, and if the task is not too difficult. Moreover, the theory of survey satisficing states that motivation, abilities, and task difficulty are not independent of each other, which provides an explanation for inconsistent findings in the literature. Results from two egocentric network studies study among German adults $(N=756)$ and among Dutch middle school students $(N=679)$, in which network contacts were also interviewed, are in line with these hypotheses. Design recommendations for egocentric network studies are provided.
\end{abstract}

\section{Introduction}

Proxy questions play a central role in egocentric network studies in which respondents (egos) are first asked to name social contacts (alters) and, subsequently, are asked to provide information about these contacts (Marsden, 1990). The latter takes place in "name interpreter questions" in which egos give proxy reports about their alters' characteristics (e.g., socioeconomic traits, their attitudes, and behavior) so that it is not necessary to interview the alters as well (Marsden, 2011; Perry et al., 2018; Stark, 2018). Proxy reports are also widely used in general population surveys such as the U.S. Census, the U.S. Current Population Survey, or the British Labour Force Survey. In these studies, one household member is interviewed who then provides proxy reports about the other members of the household. This approach allows for a quicker collection of the relevant information and is less expensive than interviewing every household member because interviewers need not come back to the same household multiple times (Cobb, 2018). Market research makes use of proxy reports for the same reasons (Bickart et al., 2006; Menon et al., 1995). Furthermore, medical researchers frequently use proxy responses to gather information on patients' health when the patients are no longer or not yet able to express themselves (Pearcy et al., 2008; Roydhouse and Wilson, 2017).

Given the wide use of proxy reports, it is of great importance to understand under which conditions answers given by egos about characteristics of their alters can adequately replace directly asking these alters. Typically, researchers making use of proxy reports are less concerned with the accuracy of the proxy report compared to an objective gold standard (but see Cobb, 2018; Magaziner et al., 1997) but with the question to what extent proxy questions can be used to replace alters' self-reports. Previous research has therefore focused primarily on congruence, or agreement, between proxy reports of ego respondents and self-reports of their alters about the alters' characteristics (e.g., Dawe and Knight, 1997; Green et al., 2014; Jäger, 2005; Roydhouse and Wilson, 2017).

This work on proxy reports about alters' characteristics relates to a long tradition of research on informant accuracy in egos' perception of the structure of social networks (Brands, 2013; Brashears and Quintane, 2015). Researchers sometimes compare these perceptions to an objectively determined social structure (e.g., Brashears et al., 2016; Butts, 2003; Freeman et al., 1987) but more often assess the congruence in participants' reports of social relationships (e.g., Krackhardt and Kilduff, 1999; Lee and Butts, 2020). Similar to research on proxy reports about alters' characteristics, much of this work on cognitive social structures has predicted congruence between egos' and alters' perception of the network structure by egos' characteristics such as their motivation (Casciaro, 1998), their power and status (Marineau et al., 2018; Simpson

\footnotetext{
* Corresponding author.

E-mail address: t.h.stark@uu.nl (T.H. Stark).
} 
et al., 2011), as well as characteristics of the relationships between ego and alter, such as their closeness (Krackhardt and Kilduff, 1999).

Research on the congruence between egos' proxy reports about their alters' characteristics and self-reports of these alters has produced some important insights. For instance, proxy reports about observable characteristics such as alters' gender or ethnicity tend to be more in agreement with alters' answers than proxy reports about emotions or attitudes (Green et al., 2014; Matza et al., 2004; White and Watkins, 2000). However, the literature is also marked by many inconsistent findings (cf. Roydhouse and Wilson, 2017). Some studies found, for example, egos' proxy reports to be more in line with alters' self-reports, the more frequently ego and alter had contact, the closer their relationship was, or the more they had discussed the topic of the proxy question in the past (e.g., Bickart et al., 2006; Jäger, 2005; Pearcy et al., 2008). Other investigations did, however, not find evidence in line with these ideas (e.g., Dawe and Knight, 1997; White and Watkins, 2000).

The present study proposes the theory of survey satisficing (Krosnick, 1999, 1991) as a theoretical framework to understand which characteristics of egos and of the survey contribute to the congruence between egos' proxy reports and alters' self-reports. This theory states that survey respondents' motivation, their ability to answer a question, and the difficulty of the question determine the quality of an answer. Congruence should thus be higher if egos are motivated to participate in the study and to remember information about their alters, if they possess the ability to accurately respond to the proxy question, and if the information asked for in the proxy question is easily accessible. Moreover, the theory of survey satisficing states that motivation, ability, and task difficulty are not independent but instead moderate each other's effects. For instance, a highly motivated ego may not be able to accurately report alter's attitudes if the relationship between ego and alter is not close enough for ego to actually know alter's attitudes. The present study will examine whether the interdependence between motivation, ability, and task difficulty might offer an explanation for the inconsistent findings about congruence in proxy reports.

\subsection{Proxy reports and the theory of survey satisficing}

Among survey methodologists, it is now widely believed that the quality of a response to a survey question depends on the effort a respondent is able or willing to put into the cognitive steps necessary to come up with an answer (Krosnick and Presser, 2010; Tourangeau et al., 2000). These steps involve (1) understanding the intent of the questions, (2) retrieving relevant information from memory, (3) integrating this information into a summary judgment, and (4) translating this judgment onto the answer options offered. The theory of survey satisficing argues that respondents only engage with each of these steps effortfully if three conditions are met: the respondent has sufficient motivation to answer the question, he/she has the ability to do so, and the task at hand is not too difficult (Krosnick, 1999, 1991). If the conditions are not met, respondents may skip the two middle steps in the cognitive process and use situational cues or simple heuristics to come up with an answer. This superficial way of engaging with a question ("satisficing") often leads to sub-optimal answers. The consequences are response biases that range from "don't know" responses to simply agreeing with an assertion or even mental coin flipping (Krosnick and Presser, 2010; Narayan and Krosnick, 1996).

Proxy reports in social network surveys are considerably different from standard survey questions for which the theory of survey satisficing was developed. It is reasonable to assume that respondents possess the relevant information about their own behavior or attitudes that is asked for in most standard survey questions; e.g., whether they voted in the past election or their political ideology. Satisficing, accordingly, concerns respondents' ability and motivation to retrieve the available information and the difficulty of this retrieval process. In contrast, proxy questions that are used in egocentric network surveys concern information about someone else. Not only does this increases the difficulty of the retrieval process but also the availability of sufficient information about alters' characteristics is affected by egos' ability and motivation to store the information in their memory in the first place. Accordingly, incongruent answers may not only be a result of satisficing during a social network survey but also of satisficing when egos could learn relevant information about their network contacts that are asked for in the survey.

If one is interested in how people are affected by the cognitive representation of their social networks, the question of whether the perceived information about network contacts align with their selfreports is less relevant (Krackhardt, 1987). However, because incongruent proxy reports might also represent measurement errors caused by incomplete memory storage and retrieval, attempts to reduce this bias in the data remain important.

The theory of survey satisficing offers a theoretical framework to organize and understand previous findings on the congruence between egos' proxy reports and alters' self-reports in network studies. Without explicitly linking it to the theory of survey satisficing, past research has expected higher levels of agreement if ego respondents possessed sufficient motivation, had the necessary abilities to answer a proxy question, and faced an easy task.

\subsubsection{Motivation}

The theory of survey satisficing argues that respondents need sufficient motivation to invest the cognitive effort necessary to answer a survey question. This motivation can be situational, limited to the interview, and mainly affect the retrieval process of relevant information. For instance, motivation is higher the more respondents feel accountable for their answers (Tetlock, 1983) and the earlier a question is asked in a questionnaire because respondents experience fatigue over time (Krosnick et al., 2002). Motivation can also relate to people's characteristics and affect both the likelihood to store and retrieve information asked for in a proxy question. For instance, motivation increases with the relevance people attribute to the topic of a question (Krosnick, 1991) and motivation is higher the more people possess personality traits that give them intrinsic rewards from mental exercises, such as need for cognition (Cacioppo and Petty, 1982). Motivation is thus specific to the ego respondent in a network study and does not depend on characteristics of the networks or relationships in the network.

In line with the idea that egos' motivation increases congruence between egos' proxy reports and alters' self-reports, medical studies expected that more severe illnesses of children would motivate their parents to give accurate accounts of their children's welfare (Roydhouse and Wilson, 2017). However, an overview study reports mixed findings of studies comparing self-reports of child patients and proxy reports of their parents (Matza et al., 2004). Sudman et al. (1994) argued that the relevance a respondent attributes to the question topic should increase agreement. However, their empirical study did not confirm this expectation (see also Jäger, 2005).

\subsubsection{Ability}

Survey satisficing theory suggests that respondents' ability depends on the knowledge they possess about what is asked in a survey question (Krosnick, 1991) and on their cognitive sophistication, which helps to recall the information needed to generate an optimal answer (Holbrook et al., 2007; Krosnick et al., 2002; Narayan and Krosnick, 1996; Peytchev, 2009). Applied to social network studies, this suggests that congruence should be higher the more egos know about their alters and that cognitive sophistication may help not only with retrieval of relevant information but also with storing information about network contacts.

In fact, most previous research on the congruence of proxy reports and alters' self-reports focused on factors that might increase egos' knowledge of alters' attitudes and behavior. Egos were more likely to give the same answers as their alters when they participated in the behavior that was asked for in the proxy question (Menon et al., 1995; 
Sudman et al., 1994). Some studies found higher congruence the more often ego and alter had discussed the topic of the proxy question (Bickart et al., 2006; Menon et al., 1995), but others found no such relationship (Jäger, 2005). Some studies found more agreement the more frequently ego had contact with alter (Jäger, 2005), but other studies reported no such effect (Schenk, 1995; White and Watkins, 2000). Some studies found higher congruence the closer the relationship between ego and alter was (Harling et al., 2015; Pearcy et al., 2008; Roydhouse and Wilson, 2017; Young et al., 2016), but other studies found no evidence in line with this (Dawe and Knight, 1997; Pfenning et al., 1991; White and Watkins, 2000). The duration of the relationship between ego and alter did not predict agreement in the few studies that explored this association (Harling et al., 2015; White and Watkins, 2000).

Few studies have explored egos' cognitive resources as a predictor of agreement between proxy reports and self-reports. Three studies in the medical domain tested whether higher educated respondents (as a proxy measure of cognitive sophistication, see Holbrook et al., 2007; Narayan and Krosnick, 1996; Peytchev, 2009) produce more congruent answers (see Roydhouse and Wilson, 2017). This hypothesis was, however, not confirmed. In sum, there is some evidence that the types of relationship people have with each other (for instance characterized by closeness or interaction and communication frequency) affect egos' abilities to answer proxy questions whereas egos' cognitive sophistication seems less relevant.

\subsubsection{Task difficulty}

Although proxy questions are always difficult to answer because they concern information about somebody else, the theory of survey satisficing predicts that difficulty further depends on how easily accessible the relevant information is in memory. Task difficulty is also affected by survey design choices, such as the complexity of the question wording or the complexity of the answer alternatives (Krosnick, 1999, 1991).

In line with the idea of memory accessibility, research has consistently found that egos and alters' answers were more in agreement to proxy questions about observable characteristics such as age, marital status, or educational level than to proxy questions about less observable topics such as HIV status, emotions, or attitudes (Dawe and Knight, 1997; Green et al., 2014; Magaziner et al., 1997; Matza et al., 2004; White and Watkins, 2000). Obviously, there is an information asymmetry between ego and alter in that ego is only able to correctly report non-observable characteristics of an alter if the alter shared the information at some point directly or indirectly with ego (Todorov and Kirchner, 2000).

Network research also found that proxy questions that offered ordinal answer scales led to levels of agreement that were statistically indistinguishable from random guessing (Green et al., 2014). Such answer scales may increase task difficulty as they typically leave room for interpretation, which can be particularly problematic in social network surveys. That is, both ego and alter could have the exact same attitude or behavior in mind but translate it to slightly different scale points. For instance, in an egocentric network study among people living with HIV/AIDS in Uganda, Green et al. (2014) found no significant congruence between egos' and alters' answers about their frequency of discussing HIV testing with each other when their answers were treated as ordinal variables. However, congruence was considerably higher when not only exact matches but also similar answers on a continuous scale were considered to be congruent answers. This suggests that network studies can reduce the task difficulty and increase congruence by offering response options that do not leave room for interpretation.

\subsection{Hypotheses}

Based on the theory of survey satisficing (Krosnick, 1999, 1991), we expect:

H1. The higher egos' motivation, the higher the congruence between proxy reports and alters' self-reports.

H2. The higher egos' abilities to answer the proxy question, the higher the congruence between proxy reports and alters' self-reports.

H3. The more difficult the task to answer a proxy question, the lower the congruence between proxy reports and alters' self-reports.

The theory of survey satisficing also offers a potential explanation for the inconsistent findings in the literature. The theory states that the three determinants of satisficing may not be independent but moderate each other's effect (Krosnick, 1991; Narayan and Krosnick, 1996). For instance, research found that the likelihood for response biases depended on the interaction between respondents' education (abilities) and how late in the survey a question was asked (motivation) (Krosnick et al., 2002) or on the interaction between respondents' education (abilities) and the question wording (task difficulty) (Holbrook et al., 2007). With regard to proxy questions, such interdependence between the three determinants could, for instance, mean that egos may be highly motivated to give an accurate answer but might still fail to do so if they do not possess enough knowledge about their alters. Moreover, whether egos can answer even the easiest question might depend on the type of tie they have with their alters. For instance, a work colleague may be better able than a friend to answer questions about alters' work commute. Accordingly, we expect:

H4. Motivation, ability, and task difficulty moderate each other's effect on the congruence between proxy reports and alters' self-reports.

Few studies have taken the potential interdependence of motivation, ability, and task difficulty into account. Jäger (2005) found a significant interaction between motivation and ability indicating that egos were more likely to give congruent proxy reports the more relevant they considered the topic of the question and if they had discussed the topic with alter. Two other tests of moderation in the literature concerned interactions between different measures of the same determinant of survey satisficing. Jäger (2005) found a negative interaction between two indicators of respondents' abilities, the closeness of the relationship between ego and alter and the frequency of their contact. Bickart et al. (2006) found no significant interaction between two other indicators of respondents' ability, the time they discussed the topic with their alter and whether egos counted or estimated alters' behavior.

Because this is a special issue on network data collection, we first discuss the setup of the two empirical tests of the hypotheses. The two studies use different designs to collect information on egos' perception of their network contacts and to validate these responses by interviewing the nominated alters as well. Study 1 concerns discussion and helping networks of parents of primary school children collected with face-to-face and telephone surveys in Germany. Study 2 focuses on friendship relations of 13-year-old school children assessed in a sociocentric network study in the Netherlands. We then report results of the empirical tests of the hypotheses in Study 1 and Study 2. The discussion section ends with a list of design recommendations for future egocentric network studies based on the findings of this research.

\section{Data sources and design considerations}

\subsection{Design of egocentric study 1}

Data came from a study of the educational decisions of parents of primary school children in Germany (Stocké, 2007). The target population consisted of non-immigrant German parents of children that were in one of 48 randomly selected primary schools in the south of the German federal state Rhineland-Palatinate. Of 989 children (response rate $45.2 \%$ ), one parent agreed to participate in a computer-assisted personal interview (CAPI) at their home in the fall of 2003. This was the mother in $94.4 \%$ of the cases and in $5.6 \%$ the father. We recruited and trained the interviewers ourselves because of bad experiences with a 
professional survey agency in a past project (data accuracy checks suggested falsified interviews). The decision for a personal interview at the home of the respondents was made because we expected that the face-to-face interactions would allow interviewers to build the needed trust in an interview concerning people's children. The interviews took $72 \mathrm{~min}$ on average.

Study 1 employed a traditional egocentric network design in that the egos (mothers/fathers) could freely nominate their alters in response to three name generator questions (Campbell and Lee, 1991; Marsden, 2011). Research has shown that asking more than one name generator question produces more accurate measures of the size of people's networks (Marin and Hamilton, 2007). Egos were asked to nominate (1) up to five persons with whom they discussed important personal matters (Burt, 1984), (2) up to five persons who looked after their home during vacations, and (3) up to five persons with whom they frequently spent their leisure time (Fischer, 1982). On average, 4.4 of the potentially 15 alters were nominated. Five alters were automatically chosen for proxy questions about these alters' characteristics. If fewer than five alters were nominated, proxy questions were asked about all of them. At the end of the survey, egos were asked for the telephone numbers of their alters. If egos did not want to provide the number, they were asked to ask their alters for their permission. In this case, the interviewer called egos one week after the interview to inquire about the telephone numbers. For 2,550 (59.4\%) alters, the telephone number was provided.

Follow-up computer-assisted telephone interviews (CATI) in the spring of 2004 led to 1,792 (response rate $70.3 \%$ ) interviews with alters nominated by 756 egos. For these ego-alter dyads, the proxy answers, as well as the self-reports of alter on the same questions were known. In this study, we opted for CATI interviews because the alters could live anywhere in Germany. This would have made face-to-face interviews extremely expensive. Moreover, the alter interviews were comparably short (25 min on average) and we expected high levels of cooperation since the alters were made aware that we had received their telephone numbers from ego and that ego had already participated in an interview. The high response rate and very little item nonresponse in this study showed that this expectation was correct.

\subsection{Design of egocentric study 2}

An important shortcoming of Study 1 was that the ego and alter interviews were conducted in different survey modes (face-to-face and telephone interviews). Incongruent answers could thus be a consequence of well-known mode effects (Tourangeau et al., 2000). To overcome this limitation, we collected data for Study 2 in the same survey mode. This study made use of sociocentric (whole) network data that were collected in schools. In contrast to most sociocentric studies that do not ask proxy questions, such questions were asked about a subset of students' networks at school. In this design, egos and alters were both part of the sample, which allowed comparing egos' responses to the proxy questions to the self-reports of the alters.

Data were collected as part of the secondary school module of The Arnhem School Study (TASS, Stark and Flache, 2012), a longitudinal study of students' social networks in their first years of secondary education (age 13). Data for the current study came from Wave 3, which was conducted after students had been together in their new classes for about one school year (June 2009). A total of 1,197 students participated in the data collection (participation rate $87.6 \%$ ). Per school class, all students simultaneously completed the questionnaire online on separate computers in their school's computer lab. A teacher read instructions to the students and supervised the completion of the questionnaires. Pilot tests of the survey suggested that most students were able to maintain their concentration for only about 30 min while completing a digital survey in a computer lab. This was surprising to us because younger students had no problem maintaining their concentration for $40 \mathrm{~min}$ (and longer) while completing a very similar questionnaire on paper for the primary-school module of TASS. As a consequence, the digital questionnaire was shortened to an average response time of about $30 \mathrm{~min}$.

The name generator question asked students to name their friends who were part of to a different first-year school class in the same school. In the Netherlands, students spend the whole day with the same group of about 20-30 classmates and can interact with friends in different classes only during recess or after school. Proxy questions thus concerned friends who were not present during the data collection in each school class. No proxy questions were asked in two schools of the highest academic track with a total of 178 students. Not all first-year classes in these schools participated in the data collection so that agreement between proxy and self-reports could not be assessed.

We opted for a single name generator in the interest of time and because most sociocentric studies on adolescents at the time of the data collection focused only on friendship ties (e.g., Goodreau et al., 2009). If students indicated that they had friends in other first-year classes of the same school, they were asked to enter the name of up to five friends in separate text boxes that were displayed on the same screen. We decided to allow up to five friendship nominations because students had nominated on average between 4.02 and 4.89 friends within their school classes in previous waves (Stark and Flache, 2012). We expected that they had fewer friends in other school classes. Moreover, all text boxes were shown on the same page because research has found that people tend to match the number of network contacts to the number of name boxes displayed (Vehovar et al., 2008).

In total, $781(76.6 \%)$ students nominated at least one friend about whom proxy answers could be given. On average, students nominated 2.26 friends in other school classes. While typing, a list of matching names (first name and the first letter of the last name) popped up from which the students could select their friends. The names had been provided by the schools after parents had received an information letter that offered them the opportunity to deny participation of their child (passive consent). On the next two screens, all proxy questions were asked for the first nominated friend. The procedure was then repeated for all friends that were nominated. Such alter-wise question formats have been found to increase break-offs and item-nonresponse (Vehovar et al., 2008) and reduce the reliability of responses (Coromina and Coenders, 2006). We did not experience any break-offs and very little unanswered questions. Nevertheless, future studies are advised to ask one question for all alters before moving on to the next question.

\section{Study 1}

The data for Study 1 were originally collected to compare to what extent parents' educational decisions are affected by their own educational aspirations, their perception of their social contacts' aspiration, and the actual aspiration of these contacts (Stocké, 2007). The present research reports a secondary analysis of these data.

\subsection{Variables}

\subsubsection{Proxy questions}

Seven proxy questions were asked for each alter. The first five proxy questions asked about socio-demographic characteristics: alter's occupational status (3 answer categories - see Online Appendix 1), alter's current occupation (7 answer categories), alter's highest educational degree (8 answer categories), the number of alter's children, and alter's age.

The last two questions asked about alter's attitudes toward education. Respondents were asked, "What does $<$ name alter $>$ think of the opinion that children will become more arrogant, the longer they attend 
school?" The second question asked, "How strongly would he/she agree with the opinion that education is a value on its own?" Answers to both questions could be given on 7-point Likert-type scales ranging from 1 "agrees absolutely not" to 7 "agrees completely". ${ }^{1}$ 7-point scales were chosen because research found this number of response options to produce responses with optimal validity and reliability in attitude questions (Krosnick and Presser, 2010).

A total of 12,544 proxy questions were asked (1,792 alters times 7 questions) that could be validated by asking the same questions to the respective alter. Of these, 12,161 answers (given by 1790 alters and 756 egos) can be used in the analyses due to missing values in either ego's proxy response or alter's self-report. The dependent variable indicating congruence between proxy report and alter's self-report was coded 1 if ego and alter gave the exact same answer to the same question and 0 otherwise.

\subsubsection{Task difficulty}

Proxy questions about socio-demographic characteristics (occupational status, occupation, highest degree, number of children, age) represented an easy task (coded 0) whereas questions about attitudes (education is a value by itself, education makes children become arrogant) formed a difficult task (coded 1). The underlying assumption was that socio-demographic characteristics are more easily observable (e.g. through attending birthday parties, seeing the children during a visit) than attitudes.

Attitude proxy questions that provide a Likert-type answer scale form a particularly difficult task because they are (1) about nonobservable characteristics of alter and (2) the answer scale leaves room for interpretation (see above). To separate the effect of task difficulty due to the two reasons, we generated a second dependent variable that adjusted for potential differences in the interpretation of the response options of the answer scale. This version allowed a deviation of the proxy answer from alters' self-reports by $+/-1$ to the last two proxy questions that were measured on 7-point Likert-type scales.

\subsubsection{Motivation}

Respondents' motivation was measured by five questions about the personal/societal relevance that egos attributed to education, the topic of the survey. The question wordings can be found in the Online Appendix. Answers could be given on seven-point scales, with higher values indicating more importance attributed to education. Responses to the five questions loaded clearly on one factor in a principal component factor analysis (see also Stocké, 2005). Moreover, a Cronbach's $\alpha$ of .70 indicated reasonable reliability of the scale. Answers were averaged across all five questions and coded to range from 0 to 1 , with higher values indicating more personal/societal relevance of education. It should be noted that, although topic relevance is a common operationalization of motivation according to the theory of survey satisficing (Krosnick, 1991), attributing more relevance to education could also increase the likelihood that egos discussed the topic with their alters, which ties it closely to egos' abilities.

\subsubsection{Abilities}

We expected the respondents to have more knowledge about their alter, the closer their relationship was (Pfenning et al., 1991) and the longer they knew each other (Menon et al., 1995; White and Watkins, 2000). Relationship closeness was assessed by asking "Please tell me how your relationship with $<$ name of alter $>$ can be characterized best? This person is (1) Not close to me at all - (7) Very close to me." Answers on a 7-point scale were coded to range from 0 to 1 with higher values indicating a closer relationship. Relationship length was asked as

\footnotetext{
${ }^{1}$ An additional proxy question asked which educational degree alter might aspire for ego's child. This question could not be used in the current study because alters received different response options than egos.
}

Table 1

Congruence of Ego's Proxy Reports and Alter's Self Reports in Study 1 ( $\mathrm{N}=1,790$ dyads $)$.

\begin{tabular}{|c|c|c|c|c|c|}
\hline \multirow[b]{2}{*}{ Proxy questions } & \multicolumn{2}{|c|}{ Exact overlap } & \multicolumn{2}{|c|}{$\begin{array}{l}+/-1 \text { scale point } \\
\text { difference }\end{array}$} & \multirow{2}{*}{$\begin{array}{l}\text { valid } N \\
\text { (dyads) }\end{array}$} \\
\hline & $\begin{array}{l}\% \\
\text { congruence }\end{array}$ & Kappa & $\begin{array}{l}\% \\
\text { congruence }\end{array}$ & Kappa & \\
\hline $\begin{array}{l}\text { Occupational } \\
\text { status }\end{array}$ & 86.0 & $.72^{* * *}$ & - & & 1,755 \\
\hline Occupation & 79.7 & $.59^{* * *}$ & - & & 1,748 \\
\hline Education & 73.0 & $.62^{* * *}$ & - & & 1,639 \\
\hline $\mathrm{N}$ of children & 94.5 & $.92^{* \cdots *}$ & - & & 1,756 \\
\hline Age & 58.4 & $.57^{* \cdots *}$ & - & & 1,744 \\
\hline $\begin{array}{l}\text { Children } \\
\text { become } \\
\text { arrogant }\end{array}$ & 41.4 & $.04^{* *}$ & 66.2 & $.45^{* * *}$ & 1,752 \\
\hline $\begin{array}{l}\text { Value on its } \\
\text { own }\end{array}$ & 46.5 & $.05^{* *}$ & 79.0 & $.60^{* * *}$ & 1,767 \\
\hline
\end{tabular}

"Could you please tell me for how many years you have known $<$ name of alter $>$ personally?" To avoid artifacts caused by outliers, we first recoded $0.56 \%$ of cases, which indicated a relationship length of more than 45 years, to a value of 45 . The scale was then transformed to range from 0 to 1 with 1 indicating 45 years. Relationship closeness and relationship length correlated at $r=.43(p<.001)$. To increase reliability, we averaged these subjective indicators of ego's perception of the relationship to form an index of the ego-alter relationship quality.

Cognitive sophistication measured in years of egos' education constituted an objective measure of respondents' abilities (Holbrook et al., 2007; Narayan and Krosnick, 1996; Peytchev, 2009). In the Online Appendix, it is explained how the German educational degrees were translated into years of education and how these degrees were distributed in the present sample. The final scale was transformed to range from 0 to 1 with 0 indicating the minimum of 7 years of education in the sample and 1 representing a university degree.

\subsection{Analysis}

The congruence of proxy answers and alters' self-reports was first assessed with Cohen's kappa. We then predicted agreement between ego and alter using logistic multilevel modeling. Responses to all seven proxy questions were analyzed simultaneously. Three-level multilevel models were set up in which the congruence of each of the seven proxy questions was nested in 1,790 ego-alter dyads that were nested in 765 egos. In logistic multilevel models, the variance on the lowest level (here, the proxy questions) is fixed to a constant (Snijders and Bosker, 1999, p. 220). A Wald test was used to test for significant variance at the higher levels. To reduce problems due to multicollinearity, all continuous variables were centered on the sample mean in the multilevel models (Snijders and Bosker, 1999).

\subsection{Results}

There was a large amount of agreement in the proxy reports and alters' self-reports. Among the observable characteristics, congruence ranged from $58.4 \%$ (Cohen's kappa $\kappa=.57, p<.001$ ) for the age of alter to $94.5 \%(\kappa=.92, p<.001)$ for the number of alter's children (Table 1). Congruence was considerably lower for the non-observable attitude questions (41.4\% agreement, $\kappa=.04, p=.005$ and $46.5 \%$ 
agreement, $\kappa=.05, p=.004) .^{2}$ The amount of agreement increased considerably for these questions when we allowed for $a+/-1$ scale point difference between proxy report and self-report to adjust for potential differences in the interpretation of the answer scale. Congruence rose to $66.2 \%(\kappa=.45, p<.001)$ and $79.0 \%(\kappa=.60, p<.001)$, making it more similar to the congruence for observable characteristics (Table 1). This suggests that the difficulty of the attitude proxy questions was mainly due to the use of Likert-type scales and to a lesser extent due to being about non-observable characteristics.

Across all 12,161 proxy responses, the mean congruence was 0.68 , indicating that just under $70 \%$ of all proxy reports exactly matched alters' self-reports (see Table B1 in the Online Appendix for descriptive statistics for all variables). When we allowed for $+/-1$ scale point difference in the non-observable attitude proxy questions, the mean congruence was 0.77 .

Multilevel models showed that all determinants of survey satisficing were (marginally) significant predictors of congruence between proxy reports and alters' self-reports when we only predicted exact matches of the answers (Model 1 in Table 2). Agreement between ego and alter was more likely, the more personal/societal relevance egos attributed to the topic of the survey, the closer the relationship between ego and alter, and the higher educated ego was, though this latter effect was not significant at the conventional level. In line with previous research, task difficulty turned out to be the strongest predictor. There was significantly less congruence in attitude proxy questions than in proxy questions about observable characteristics. All of these findings are in line with Hypotheses 1-3, indicating that respondents' motivation, ability, and the task difficulty predict congruence in proxy reports and alters' self-reports.

Significant interactions between some of the three determinants of survey satisficing indicated that these predictors were not independent of each other. The interaction between motivation and task difficulty was significant and positive $(b=.90, p=.003$, Model 2 in Table 2), indicating that higher motivation could compensate to some extent for the increased difficulty of answering an attitude proxy question. The conditional main effect of the relevance index was no longer significant, which suggests that egos' motivation mattered for the congruence of difficult proxy questions but less so for easy questions (see Fig. $\mathrm{C} 1$ in the Online Appendix for a predicted-values plot of this interaction). Also the interaction between task difficulty and egos' abilities was significant but negative $(b=-.39, p=.046)$. Given the positive and highly significant conditional main effect of the relationship index $(b=.53, p<.001)$, the negative interaction suggests that higher abilities in terms of closeness to the alters helps with easy but less so with difficult proxy questions (see also Fig. C2 in the Online Appendix).

The negative interaction between task difficulty and abilities did not reemerge when abilities were measured with years of education (cognitive sophistication, Model 3 in Table 2). The interaction between egos' motivation and their abilities was never a significant predictor of congruence, independently of whether it was measured through the relationship index $(b=-.44, p=.528$, Model 2$)$ or education $(b=.48, p$ $=.499$, Model 3). Accordingly, the evidence was only partially in line with Hypothesis 4.

Similar results emerged when we allowed for $a+/-1$ scale point difference in the proxy reports of the two attitude questions compared to alters' self-reports (see Models 4-6 in Table 2). This suggests that proxy questions about non-observable attitudes are particularly difficult to be answered congruently, even if the answer scale allows for some tolerance in how it is interpreted. Congruence is significantly higher if ego and alter have a closer relationship, but this closeness cannot

\footnotetext{
${ }^{2}$ Note that the original responses by egos and alters to the attitude questions were strongly skewed (skewness varied between 1.42 and 2.26). Since Cohen's kappa is sensitive to the distribution of variables, the low kappa potentially underestimates the relationship between the variables.
}

compensate for the added difficulty of attitude proxy questions.

\subsection{Discussion of study 1}

Results of Study 1 were largely in line with the theory of survey satisficing (Krosnick, 1999, 1991). Proxy reports and alters' self-reports were more likely to be congruent if egos had higher motivation, possessed more abilities by having a closer tie with their alters, and when the task was not too difficult. Moreover, moderation analyses indicated that some of these factors were not independent predictors. Motivation turned out to compensate to some extent for the added difficulty of attitude proxy questions. Interestingly, moderation of the subjective indicator of egos' abilities, the relationship closeness, did not fit this pattern. A closer relationship between ego and alter increased the likelihood of congruent answers mainly for observable proxy questions but less so for the more difficult attitude questions.

Study 1 suffers from two important shortcomings. First, the difficult proxy questions were not only about non-observable attitudes but, as it is common for attitude questions, also measured with Likert-type answer scales that left room for interpretation. This was different from the easy proxy questions that asked about observable characteristics and mainly offered clearly labeled categorical answer scales. This double penalty of difficult proxy questions may have caused the particularly strong impact of task difficulty on the congruence. Second, the measure of motivation may have been confounded with respondents' ability to answer proxy questions. Egos who attributed more relevance to education were perhaps more likely to discuss this topic with their alters, increasing their abilities to answer proxy questions about alters' attitudes toward education. It is thus impossible to conclude from these data that increased motivation always compensates for the increased difficulty of answering an attitude proxy question.

To address these shortcomings, the hypotheses were also tested in a study that manipulated egos' motivation by asking network questions early or late in the survey and in which difficult and easy proxy questions were measured with the same answer scale so that the only difference was whether egos' were asked proxy questions about observable characteristics of their alters or not.

\section{Study 2}

\subsection{Variables}

\subsubsection{Proxy questions}

The first three proxy questions in the school study asked for the friends' music taste. Students were asked, "What do you think does < name of friend $>$ think of the following music styles?" Categories were displayed as "Dutch pop music (e.g., Jan Smit, Nick and Simon)," "Dutch hip hop (e.g., Yes-R, Ali B.)," and "English Pop (e.g., Duffy, Robbie Williams)." Answers could be given on five-point scales ranging from 1 "thinks it is very stupid" to 5 "thinks it is very good."

The next three proxy questions were introduced with the question "How much, do you think, does < name of friend > like the following?" Categories were "hanging out on the street," "going to school," and "wearing branded clothes." Answers were given on five-point scales ranging from 1 "not at all" to 5 "very much." At another position in the questionnaire, students were asked to answer the same questions about themselves. These answers were used as alters' self-reports if the students were nominated as a friend. Congruence between proxy answer and self-report was coded 1 if ego and alter gave the exact same answer and 0 otherwise.

\subsubsection{Motivation}

Motivation was experimentally manipulated by randomly assigning students to either answer the proxy questions early in the questionnaire (after approx. $5 \mathrm{~min}$ ) or toward the end (after approx. $25 \mathrm{~min}$ ). We assumed that students were more fatigued at the end of the 
Table 2

Predicting Congruence between Ego's Proxy Answer and Alter's Self-Report in Study 1.

\begin{tabular}{|c|c|c|c|c|c|c|c|c|c|c|c|c|c|c|c|c|c|c|}
\hline \multirow[b]{3}{*}{ Predictors } & \multirow{2}{*}{\multicolumn{3}{|c|}{$\begin{array}{l}\text { Exact match } \\
\text { Model } 1\end{array}$}} & \multirow{2}{*}{\multicolumn{3}{|c|}{ Model 2}} & \multirow{2}{*}{\multicolumn{3}{|c|}{ Model 3}} & \multicolumn{9}{|c|}{$+/-1$ scale point difference in attitude proxy questions } \\
\hline & & & & & & & & & & \multicolumn{3}{|l|}{ Model 4} & \multicolumn{3}{|l|}{ Model 5} & \multicolumn{3}{|l|}{ Model 6} \\
\hline & $b$ & s.e. & OR & $b$ & s.e. & OR & $b$ & s.e. & OR & $b$ & s.e. & OR & $b$ & s.e. & OR & $b$ & s.e. & OR \\
\hline Intercept & $1.31^{* * *}$ & .03 & & $1.31^{* * *}$ & .03 & & $1.30^{* * \ldots}$ & .03 & & $1.31^{* * *}$ & .03 & & $1.32^{* * *}$ & .03 & & $1.31^{* * *}$ & .03 & \\
\hline \multicolumn{19}{|l|}{ Motivation } \\
\hline $\begin{array}{l}\text { Relevance index }{ }^{\mathrm{a}} \\
\text { Abilities }\end{array}$ & $.52^{* *}$ & .16 & 1.69 & .20 & .19 & 1.22 & .26 & .20 & 1.29 & $.45^{* *}$ & .17 & 1.56 & .20 & .20 & 1.22 & .27 & .20 & 1.31 \\
\hline Relationship index ${ }^{a}$ & $.39^{* * * *}$ & .10 & 1.47 & $.53^{* * *}$ & .12 & 1.70 & $.38^{* * * k}$ & .10 & 1.47 & $.29^{* *}$ & .10 & 1.34 & $.54^{* * * *}$ & .12 & 1.72 & .29 ** & .10 & 1.34 \\
\hline Education in years ${ }^{\mathrm{a}}$ & $.19^{\dagger}$ & .10 & 1.21 & $.19^{\dagger}$ & .10 & 1.21 & .10 & .12 & 1.10 & $.21 *$ & .10 & 1.24 & $.21 *$ & .10 & 1.24 & .10 & .12 & 1.10 \\
\hline \multicolumn{19}{|l|}{ Task difficulty } \\
\hline Question type attitude & $-1.56^{* * *}$ & .04 & 0.21 & $-1.56^{* * *}$ & .04 & 0.21 & $-1.56^{* * *}$ & .04 & 0.21 & $-.32^{* * *}$ & .05 & 0.73 & $-.32^{* * *}$ & .05 & 0.73 & $-.31^{* * *}$ & .05 & 0.73 \\
\hline \multicolumn{18}{|l|}{ Two-way interactions } & \\
\hline $\begin{array}{l}\text { Motivation * abilities } \\
\text { (relationship index) }\end{array}$ & & & & -.94 & $\begin{array}{l}.30 \\
.70\end{array}$ & $\begin{array}{l}2.46 \\
0.64\end{array}$ & $.79 \%$ & .31 & 2.21 & & & & $\begin{array}{l}.77^{*} \\
-.95\end{array}$ & $\begin{array}{l}.32 \\
.72\end{array}$ & $\begin{array}{l}2.15 \\
0.39\end{array}$ & .60 & .32 & \\
\hline $\begin{array}{l}\text { T. difficulty * abilities } \\
\text { (relationship index) }\end{array}$ & & & & $-.39 *$ & .20 & 0.67 & & & & & & & $-.78^{* * *}$ & .21 & 0.46 & & & \\
\hline $\begin{array}{l}\text { Motivation * abilities } \\
\text { (education) }\end{array}$ & & & & & & & .48 & .71 & 1.62 & & & & & & & .31 & .74 & 1.37 \\
\hline $\begin{array}{l}\text { T. difficulty * abilities } \\
\text { (education) }\end{array}$ & & & & & & & .22 & .19 & 1.24 & & & & & & & .34 & .20 & 1.40 \\
\hline \multicolumn{19}{|l|}{ Random effects } \\
\hline - Level 3 (egos) & $.06 *$ & & & $.06 *$ & & & $.06 *$ & & & $.06 *$ & & & $.06^{* * *}$ & & & $.06^{* *}$ & & \\
\hline - Level 2 (alters) & .00 & & & .00 & & & .02 & & & .02 & & & .02 & & & .02 & & \\
\hline Deviance difference $\mathrm{b}^{\mathrm{b}}$ & $1371.6^{\ldots \ldots *}$ & & & $12.93^{\text {** }}$ & & & $10.34 *$ & & & 69.19 *** & & & $19.92^{* * *}$ & & & $8.00^{*}$ & & \\
\hline
\end{tabular}

Note: Total $N=12,161$ proxy answers cross-nested in 1,790 alters, nested in 756 egos.

${ }^{a}$ Continuous variables were centered in these analyses.

${ }^{\mathrm{b}}$ Log-likelihood tests with an empty model that contained no covariates (for Models 1 and 4) or with the model without interactions (for Models 2, 3, 5, 6).

${ }_{* * * *}^{* * *} p<.001$

$p<.01$.

$p<.05$

${ }^{\dagger} p<.1$ (two-tailed test) 
Table 3

Congruence of Ego's Proxy Reports and Alter's Self Reports in Study 2 ( $\mathrm{N}=1,526$ dyads).

\begin{tabular}{llll}
\hline & \multicolumn{2}{l}{ Exact overlap } & \multirow{2}{*}{ valid $N$ (dyads) } \\
\cline { 2 - 3 } Proxy questions & \% congruence & Kappa & \\
\hline Likes Dutch pop music & 28.9 & $.05^{* * *}$ & 1,339 \\
Likes Dutch hip hop music & 29.6 & $.06^{* * *}$ & 1,346 \\
Likes English pop music & 27.3 & $.06^{* * *}$ & 1,281 \\
Likes going to school & 29.9 & $.07^{* * *}$ & 1,391 \\
Likes hanging out on street & 30.8 & $.08^{* * *}$ & 1,382 \\
Likes wearing branded clothes & 36.1 & $.08^{* * *}$ & 1,396 \\
\hline
\end{tabular}

${ }_{* * * *}^{*} p<.001$ (two-tailed test).

questionnaire and thus less motivated (Krosnick, 1991; Krosnick et al., 2002).

\subsubsection{Abilities}

Study 2 also contained objective and subjective measures of students' abilities. Years of education, as used in Study 1 and in earlier research (Holbrook et al., 2007; Narayan and Krosnick, 1996; Peytchev, 2009), was not a meaningful measure of participants' cognitive sophistication because all participants were in the same year in school. Instead, the objective indicator of students' ability (cognitive sophistication) was a dummy variable indicating whether the class of a student belonged to the lowest (coded 0 ) or a higher academic track (coded 1) in the Dutch secondary school system. ${ }^{3}$ The first subjective measure was a relationship index measuring the length and closeness of the friendship. For each of the alters, ego was asked: "how long do you know $<$ name of friend >?" Numeric answers could be typed in a text box that said: "[] years." Open answers larger than 13 were set to 13 (the average age in the sample) and the scale was recoded to range from 0 to 1 . The second question of relationship quality asked, "how good of friends are you with < name of friend $>$ ?" Answers were code 0 "acquaintances," 0.33 "just friends," 0.66 "good friends," and 1 "best friends." The relationship index was created by taking the average of both scales so that higher values indicate a closer relationship.

Reciprocity of the friendship nomination formed the second subjective indicator of the relationship closeness and thus of students' abilities. Reciprocity was code 1 when alter also nominated ego as a friend and 0 otherwise.

\subsubsection{Task difficulty}

Proxy questions about observable behavior (hanging out on the street and wearing branded clothes) represented an easy task (coded 0) whereas questions about attitudes (liking of Dutch Pop, Dutch hip hop, English pop, and going to school) formed a difficult task (coded 1).

\subsection{Analysis}

Congruence of proxy answers and alters' self-reports was again assessed with Cohen's Kappa and predicted with logistic multilevel modeling. For 85 students, the agreement of proxy and self-reports could not be assessed due to missing values either in the proxy report or alter's answer. Five additional cases were removed due to missing values on the relationship closeness index. Four-level multilevel models were set up in which the congruence of each of the six proxy questions was crossnested within 719 alters and 1526 dyads, who were nested in 679

\footnotetext{
${ }^{3}$ Students are separated into three tracks in the Netherlands, the lowest (VMBO, preparatory secondary vocational education), the middle (HAVO, senior general secondary education), and the highest (VWO, pre-university education) educational track. In the available sample, $39.9 \%$ of students were in the lowest track, $46.4 \%$ were in classes of the middle track, and $14 \%$ were in the highest track. These latter two groups were combined so that the variable differentiates between the lowest track and higher tracks.
}

egos. The proxy questions were cross-nested in the alters and dyads because the same alter could be nominated by multiple egos and because alter could also have nominated ego. The nesting of egos in school classes was not accounted for in the analysis because there was no significant variance on the class level $\left(\chi^{2}(1)=0.00, p=1\right)$. The continuous relationship index was centered on the sample mean in the multilevel models.

\subsection{Results}

The congruence between proxy reports and alters' self-reports did not vary much between the six questions asked. Table 3 shows that between $27.3 \%$ (English pop music) and $36.1 \%$ (wears branded clothes) of proxy answers were equal to alters' responses. Accordingly, Cohen's Kappa was very low and varied between $\kappa=.05$ and $\kappa=.08$ (all $p<.001)$.

Across all 8,135 proxy answers, the mean congruence (exact matches) was $30.5 \%$ (see Table B2 in the Online Appendix for descriptive statistics for all variables). The randomization allocated 51.7 $\%$ of the students to receive proxy questions early in the survey when the motivation to participate should have been high. $60.1 \%$ of the students were in a class of the higher educational tracks and $30.5 \%$ of all friendship nominations in the sample were reciprocated.

Results of the main effects of ability, motivation, and task difficulty predicting congruence of proxy reports and alters' self-reports resembled those of Study 1 . Asking the question early was associated with a higher likelihood of congruent answers (Model 1 in Table 4). This was in line with Hypothesis 1. Likewise, and in line with Hypothesis 3, asking a difficult question about non-observable attitudes was associated with a lower likelihood for congruent reports. Being in the higher academic tracks was not associated with more congruent answers. However, the significant coefficient for the relationship index shows that congruence was higher for egos that reported to have a closer and longer-lasting relationship with their alters. This coefficient was reduced in size and turned insignificant when the indicator for reciprocity was added to the model (Model 2 in Table 4). This suggests that closeness and reciprocation of the friendship were closely related. Reciprocity remained a significant predictor of congruence between ego and alter. Hypothesis 2 was thus only supported for the subjective but not the objective indicators of participants' abilities.

A negative and marginally significant interaction between egos' motivation and task difficulty $(b=-.17, p=.093$, Model 3 in Table 4) together with the conditional main effects suggests that motivation increased the likelihood for congruent answers; and that this was not true for difficult proxy questions (see Fig. C3 in the Online Appendix for a predicted-values plot of this interaction). None of the other interactions was significant. This was true for both subjective indicators of students' abilities, the relationship index (Model 3) and reciprocity (Model 4), as well as for the objective indicator, the academic level of the school class (interaction with task difficulty: $b=-.10$, s.e. $=.11, p=$ .330 ; interaction with motivation: $b=-.03$, s.e. $=.11, p=.812$; results not in the table).

In the last step, we explored whether the effect of egos' perception of their relationship closeness depended on their alters' perception of the relationship closeness. This analysis was possible among those ego-alter dyads in which alter had reciprocated the friendship nomination $(n=$ 462). If egos misperceived their relationship with their alters, they may be more likely to misperceive characteristics of their alters as well. To test this possibility, an interaction was added between egos' perception of the relationship (relationship index) and alters' perception of the same relationship. Neither of the conditional main effects nor the interaction $(b=.55$, s.e. $=1.00, p=.591)$ was statistically significant. This suggests that congruence was not related to a (mis)match between egos' and alters' perception of their relationship closeness. 
Table 4

Predicting Congruence between Ego's Proxy Answer and Alter's Self-Report in Study 2.

\begin{tabular}{|c|c|c|c|c|c|c|c|c|c|c|c|c|}
\hline \multirow[b]{2}{*}{ Predictors } & \multicolumn{3}{|l|}{ Model 1} & \multicolumn{3}{|l|}{ Model 2} & \multicolumn{3}{|l|}{ Model 3} & \multicolumn{3}{|l|}{ Model 4} \\
\hline & $b$ & s.e. & OR & $b$ & s.e. & OR & $b$ & s.e. & OR & $b$ & s.e. & OR \\
\hline Intercept & $-.81^{\text {*** }}$ & .06 & & $-.86^{* k *}$ & .07 & & $-.86^{* * *}$ & .07 & & $-.97^{\star * *}$ & .08 & \\
\hline \multicolumn{13}{|l|}{ Motivation } \\
\hline Questions asked early & $.12^{*}$ & .05 & 1.13 & $.13^{*}$ & .05 & 1.13 & $.23^{*}$ & .06 & 1.26 & $.23^{*}$ & .06 & 1.31 \\
\hline \multicolumn{13}{|l|}{ Abilities } \\
\hline Higher academic level & .03 & .06 & 1.03 & .04 & .06 & 1.05 & .03 & .06 & 1.03 & .05 & .06 & 1.05 \\
\hline Relationship index (ego) & $.29 *$ & .13 & 1.34 & .19 & .14 & 1.20 & .21 & .25 & 1.23 & .19 & .14 & 1.20 \\
\hline Reciprocity & & & & $.16^{*}$ & .06 & & & & & $.30^{* *}$ & .11 & 1.34 \\
\hline \multicolumn{13}{|l|}{ Task difficulty } \\
\hline Question type difficult & $-.22^{* * *}$ & .05 & 0.81 & $-.22^{* * *}$ & .05 & 0.81 & $-.12^{\dagger}$ & .07 & 0.88 & -.08 & .08 & 0.92 \\
\hline \multicolumn{13}{|l|}{ Two-way interactions } \\
\hline Motivation * $\mathrm{T}$. difficulty & & & & & & & $-.17^{\dagger}$ & .10 & 0.84 & $-.18^{\dagger}$ & .10 & 0.84 \\
\hline Motivation * abilities (relationship index) & & & & & & & .14 & .26 & 1.15 & & & \\
\hline T. difficulty * abilities (relationship index) & & & & & & & .02 & .25 & 1.02 & & & \\
\hline Motivation * abilities (reciprocity) & & & & & & & & & & -.10 & .11 & .91 \\
\hline T. difficulty * abilities (reciprocity) & & & & & & & & & & -.14 & .11 & .87 \\
\hline \multicolumn{13}{|l|}{ Random effects } \\
\hline - Level 3 (egos) & .01 & & & .01 & & & .01 & & & .01 & & \\
\hline - Level 2 (alters) & $.08^{* * *}$ & & & $.08^{* * *}$ & & & $.08^{* * *}$ & & & $.07^{* * *}$ & & \\
\hline - Level 2 (dyads) & .05 & & & .04 & & & .05 & & & .04 & & \\
\hline Deviance difference ${ }^{\mathrm{a}}$ & $28.38^{* \cdots *}$ & & & $6.56^{*}$ & & & 3.09 & & & 5.21 & & \\
\hline
\end{tabular}

Note: Total $N=8,135$ proxy answers cross-nested in 719 alters and 1,526 dyads, nested in 679 egos.

${ }^{\text {a }}$ Log-likelihood tests. Model 1: compared to an empty model that contained no covariates. Model 2 and Model 3: compared to Model 1. Model 4: compared to Model 2 .

${ }^{* * * *} p<.001$.

${ }^{* *} p<.01$.

* $p<.05$

${ }^{\dagger} p<.1$ (two-tailed test).

\subsection{Discussion of study 2}

Study 2 provided further support for predictions based on the theory of survey satisficing (Krosnick, 1999, 1991). The likelihood for congruent proxy reports was higher when the name interpreter questions were asked early in the study (higher motivation), ego and alter had a closer relationship (abilities), alter reciprocated the tie (abilities), and when the proxy questions asked about alter characteristics that egos could more easily observe (task difficulty). Furthermore, there was again some evidence for the interdependence of the three determinants of survey satisficing. Higher motivation was associated $(p<.01)$ with a higher likelihood for congruent answers in easy proxy questions, but not in difficult proxy questions. Asking proxy questions early in a network studies while egos were still motivated increased congruence for questions about observable alter characteristics but was of little help for attitude name interpreter questions.

\section{General discussion}

We argued that the theory of survey satisficing (Krosnick, 1999, 1991) offers a theoretical framework that allows predicting when proxy reports and alters' self-reports are likely congruent and that also provides a potential explanation for the inconsistent findings in the literature on bias in proxy responses. Results from two egocentric network studies, in which interviews with the nominated alters allowed comparing proxy reports and self-reports, were largely in line with predictions based on survey satisficing theory.

Congruent answers were more likely, the higher the motivation of the respondents was. Study 2 showed that congruence was more likely for proxy questions asked early in the survey than for questions asked late when respondents were more likely to experience fatigue or were bored (Krosnick et al., 2002). Moreover, in Study 1, we found that a self-reported measure in terms of the personal/societal relevance associated with the topic of the survey predicted congruence. This latter finding is not in line with previous studies that did not find personal/societal relevance predicting agreement between ego and alter
(Jäger, 2005; Sudman et al., 1994). However, the significant interaction between motivation and task difficulty in the present research offers an explanation for this finding. That is, the personal/societal relevance respondents associated with the topic education only increased the likelihood for congruent answers about difficult attitude proxy questions toward education. It did not predict the congruence of proxy answers about demographic characteristics. Previous studies did not take such an interaction into account.

There was also evidence for the importance of respondents' abilities to answer a proxy question. In line with many previous studies (Harling et al., 2015; Pearcy et al., 2008; Roydhouse and Wilson, 2017; Young et al., 2016), we found a higher likelihood for congruent answers, the closer the relationship between ego and alter was. This was true for ego's perception of the relationship closeness and if alter reciprocated the friendship (Study 2). In Study 1, the effect of closeness depended on the difficulty of the proxy questions. Having a closer relationship could not compensate for the difficulty of answering a proxy question about non-observable characteristics. This may explain why some earlier studies did not find an effect of closeness on the likelihood of congruent proxy reports (Dawe and Knight, 1997; Pfenning et al., 1991; White and Watkins, 2000).

The objective measure of respondents' abilities, their cognitive sophistication in terms of higher levels of education, was significantly associated with more congruent answers in Study 1 but not in Study 2. This inconsistency may result from education being only a very crude proxy measure of people's actual cognitive abilities. This was particularly problematic in Study 2 where education could only be measured on the classroom level and the dichotomous indicator did not allow for much variation between classes.

Task difficulty was the strongest predictor of the congruence between the proxy report and alter's self-report. Congruence was higher for observable, socio-demographic characteristics (Study 1 ) or attitudes toward observable behavior (Study 2) than toward non-observable attitudes. This is in line with many previous studies (Dawe and Knight, 1997; Green et al., 2014; Magaziner et al., 1997; Matza et al., 2004; Todorov and Kirchner, 2000; White and Watkins, 2000). 
In sum, these results are consistent with the notion that the three determinants of survey satisficing predict congruent proxy reports and that the effects of some of these determinants are interdependent. Whereas egos' motivation is not directly affected by their social networks, egos' abilities and the task difficulty are closely related to the type of relationships that are assessed in a network study. Abilities tend to be higher for close ties (e.g., reciprocated ties, closeness) and the task difficulty is lower for questions about alter characteristics that egos can easily infer from interacting with their network contacts.

\subsection{Limitations}

The central limitation of this research is the assumption that the egos are completely responsible for the potential incongruence between their own and their alters' answers. In fact, survey satisficing theory would predict that some alters satisfice as well and produce inaccurate answers, which then leads to incongruence. As in any egocentric network study with link-tracing designs (such as respondent-driven sampling of alters or snowball sampling), egos and alters' probability to satisfice should even be correlated. Education is related to survey satisficing (Holbrook et al., 2007; Krosnick et al., 2002; Narayan and Krosnick, 1996) and social networks are known to be educationally homogeneous (McPherson et al., 2001). In addition, social desirability bias in alters' responses may cause that accurate proxy reports are labeled as incongruent. Egos could be more willing to answer truthfully about a socially undesirable attitude or behavior of their alters than these alters are. Both of these limitations may have led to an underestimation of the importance of the determinants of survey satisficing for congruent proxy reports. To overcome these problems, future work could compare proxy reports to an objective measure of alters' characteristic, if such a gold standard is available (Cobb, 2018; Magaziner et al., 1997).

The use of different subjective measures of motivation, abilities, and task difficulties across the two studies has advantages and limitations. On the one hand, the consistent results of the main effects of these indicators independently of the operationalization give strong support for the claim that motivation, abilities, and task difficulty are predictive of congruence between proxy reports and alters' self-reports. On the other hand, it remains unclear whether inconsistent findings with regard to the interactions are due to the differences in the study design or, in fact, due to the different operationalizations. We are looking forward to future tests of these alternatives.

\subsection{Design recommendations for egocentric network studies}

We summarize here the general recommendations for the design of egocentric network studies that follow from our research.

\subsubsection{Motivation}

An important distinction can be drawn between topic motivation and design motivation. The theory of survey satisficing argues that respondents' interest in the topic of a survey can increase their motivation (Krosnick, 1991) and the current research found some evidence in line with this expectation in Study 1. Accordingly, we recommend considering how interesting or relevant the research topic is to participants when designing an egocentric network study. As this might often be difficult to know upfront, a more promising way to increase data quality might be to focus on design motivation instead.

There are various design decisions researchers can make to increase their participants' motivation. The present research found that proxy answers were more congruent with alters' self-reports when the proxy questions were placed early in the survey. This finding complements recent research showing that people name more contacts in name generators when they are asked early in a questionnaire (Yousefi-Nooraie et al., 2019). Early placement may be beneficial for congruence because proxy questions require quite some cognitive effort from respondents as they have to recall information about somebody else (Marsden, 2011).
As a consequence, such questions can best be asked while participants are not yet fatigue (Krosnick, 1999) or drained of their energy due to the effortful process of completing a survey (Baumeister et al., 1998).

Self-determination theory further suggests that a loss of energy can be prevented by tasks that satisfy people's needs for competence and autonomy (Ryan and Deci, 2008). Recently developed graphical interfaces for the collection of egocentric network data (e.g., Eddens and Fagan, 2018; Hogan et al., 2020; Stark and Krosnick, 2017) may satisfy these needs better than standard egocentric questionnaires as they allow participants to visually construct and engage with their own network. In line with this, such tools have been found to increase respondents' enjoyment and motivation (Stark and Krosnick, 2017). Network researchers are thus well-advised to increase their respondents' motivation during an egocentric network survey by choosing a design that is likely to keep the participants engaged and motivated to invest the needed cognitive effort to answer questions about their contacts. Accordingly, we recommend the use of graphical egocentric network interfaces in future work and to ask network questions early in a survey.

\subsubsection{Abilities}

Participants' abilities might be more important than their motivation when they are asked to answer a proxy question. Even the most motivated respondents can only give accurate accounts of their alters if they possess the necessary information about these alters. Network researchers can use one of the limitations of Study 1 to their advantage: here, it was unclear whether participants' interest in the topic of the study was a measure of their motivation or a reflection of their abilities. Respondents with a higher interest in a topic are also more likely to discuss the topic with their social contacts and may be more motivated to remember their alters' attitudes. Thus, topic motivation might also reflect participants' knowledge about their alters' attitudes and thus increase their ability to answer proxy questions. We recommend focusing future network survey on topics that are of relevance to the participants. Through this approach, respondents' motivation and their abilities may be increased simultaneously.

Name generators in egocentric network studies can also be specified to increase egos' abilities to know their alters' characteristics. For instance, the original purpose of Study 1 was to measure how educational aspirations of social contacts affect parents' educational decisions. Accordingly, it may have been better to directly ask for network contacts with whom egos discussed educational matters than to ask for people with whom they discussed important personal matters in general (Burt, 1984). Thus, we recommend using topic-specific name generators that produce networks that are more relevant for a specific research question instead of relying on generic network generator questions, simply because they are widely used.

The finding that congruence was higher in closer and reciprocated relationships gives some additional direction for the choice of name generator questions. First, name generators should focus on close relationships when determining a person's network. Just asking for friends is not enough as we found in Study 2 that congruence increased with the closeness and reciprocity of students' friendships. A better approach is perhaps to use multiple name generators (Marin and Hamilton, 2007) and to ask name interpreter questions about those contacts that are named in most name generators. Second, it can be expected (though not directly tested in this study) that congruence in proxy reports decreases with increasing size of the network. Because it is typically assumed that people have a limited capacity for close relationships (e.g., Zeggelink, 1995), closeness should decrease with every alter elicited in a name generator. Thus, we recommend name generators that do not elicit too many names if congruent proxy reports are important.

Last, there was some evidence in Study 1 that higher education is related to more congruent proxy reports. In line with satisficing theory (Holbrook et al., 2007; Krosnick et al., 2002), this could suggest that people with higher cognitive sophistication have stronger abilities to answer proxy questions. If this reasoning is correct, network researchers 
could expect to collect more congruent proxy reports when their participants are higher educated. However, more research is needed to better understand the role of education given the non-significant effects in Study 2.

\subsubsection{Task difficulty}

The most important factor seems to be the difficulty of a proxy question. The present research suggests that name interpreter questions can reasonably well replace self-reports about observable demographic characteristics. Questions about non-observable attitudes seem less suited for the use as proxy questions (c.f. Marsden, 2011). Importantly, the difficulty of a proxy question does not only matter in terms of absolute impact but also because it can qualify the effect of respondents' motivation and abilities. This leads to the recommendation to ask proxy questions about observable characteristics of alters and not about their attitudes.

Next to being about observable traits of alter, there are many more characteristics of the proxy questions itself that make a task easy or difficult. These include the complexity of the question wording and the number of response options. The low congruence for attitude proxy questions in Study 1 may thus partially be because these questions were measured with Likert-type scales that leave room for interpretations. We recommend avoiding using such response scales in name interpreter questions (see also Green et al., 2014), and instead providing clearly labeled categorical response options.

It also seems wise to limit the number of answer categories in name interpreter questions. The proxy question about alter's age in Study 1 offered many more theoretically possible response options than the proxy question about the number of alter's children although both questions were measured with the same interval scale. Accordingly, the congruence of the age question was considerably lower than that of the number of children question. To increase congruence, we recommend reducing the complexity of the response options (Marsden, 2011), for instance by asking whether alter is younger, of the same age, or older than ego.

\subsection{Cognitive social structures}

Many of the recommendations reported here should also apply to the study of informant accuracy in the perception of the network structure (e.g., Brashears et al., 2016; Butts, 2003; Freeman et al., 1987). To the extent that errors in the recall of network members and relationships among them are due to the cognitive effort involved in remembering all ties (high task difficulty), increasing participants' motivation and abilities might also increase the accuracy of their reports of alter-alter ties. For instance, research found that factors that increase people's ability to observe network interactions such as their power (Marineau et al., 2018; Simpson et al., 2011), their position in the network (Casciaro, 1998), or social distance to alters (Krackhardt and Kilduff, 1999) were related to more accurate perceptions of the network structure. Also personality traits that are typically associated with people's motivation have been found to increase accuracy in network perceptions (Casciaro, 1998).

To sum up, the present research suggests that the theory of survey satisficing can provide helpful guidelines for the design of egocentric network studies. To improve the quality of proxy reports and perhaps also the accuracy of the network perception, researchers should consider how they can increase their participants' motivation and abilities through the research design and how task difficulty can be minimized.

\section{Acknowledgements}

This work was supported by a grant of the German Science Foundation (DFG) to the Sonderforschungsbereich 504 at the University of Mannheim and the Netherlands Organisation for Scientific Research (NWO) under the Innovational Research Incentive Scheme (VENI grant 451-14-003). The authors have no competing interests to declare
We would like to thank Jon Krosnick, Henning Silber, Marijtje van Duijn, Michael Mäs, and Andreas Flache for valuable comments and suggestions.

\section{Appendix A. Supplementary data}

Supplementary material related to this article can be found, in the online version, at doi:https://doi.org/10.1016/j.socnet.2021.01.007.

\section{References}

Baumeister, R.F., Bratslavsky, E., Muraven, M., Tice, D.M., 1998. Ego depletion: is the active self a limited resource? J. Pers. Soc. Psychol. 74, 1252-1265. https://doi.org/ 10.1037/0022-3514.74.5.1252.

Bickart, B.A., Phillips, J.M., Blair, J., 2006. The effects of discussion and question wording on self and proxy reports of behavioral frequencies. Mark. Lett. 17, 167-180.

Brands, R.A., 2013. Cognitive social structures in social network research: a review. J. Organ. Behav. 34, S82-S103. https://doi.org/10.1002/job.1890.

Brashears, M.E., Quintane, E., 2015. The microstructures of network recall: how social networks are encoded and represented in human memory. Soc. Networks 41 , 113-126. https://doi.org/10.1016/j.socnet.2014.11.003.

Brashears, M.E., Hoagland, E., Quintane, E., 2016. Sex and network recall accuracy. Soc. Networks 44, 74-84. https://doi.org/10.1016/j.socnet.2015.06.002.

Burt, R.S., 1984. Network items and the general social survey. Soc. Networks 6, 293-339.

Butts, C.T., 2003. Network inference, error, and informant (in)accuracy: a Bayesian approach. Soc. Networks 25, 103-140. https://doi.org/10.1016/S0378-8733(02) 00038-2.

Cacioppo, J.T., Petty, R.E., 1982. The need for cognition. J. Pers. Soc. Psychol. 42, $116-131$.

Campbell, K.E., Lee, B.A., 1991. Name generators in surveys of personal networks. Soc. Networks 13, 203-221.

Casciaro, T., 1998. Seeing things clearly: social structure, personality, and accuracy in social network perception. Soc. Networks 20, 331-351. https://doi.org/10.1016/ S0378-8733(98)00008-2.

Cobb, C., 2018. Answering for someone else: proxy reporting in survey research. In: Vannette, D.L., Krosnick, J.A. (Eds.), Eds.), The Palgrave Handbook of Survey Research. Palgrave Macmillan, Cham, Switzerland, pp. 87-93.

Coromina, L., Coenders, G., 2006. Reliability and validity of egocentered network data collected via web - A meta-analysis of multilevel multitrait, multimethod studies. Soc. Networks 28, 209-231. https://doi.org/10.1016/J.Socnet.2005.07.006.

Dawe, F., Knight, I., 1997. A study of proxy response on the labor force survey. Surv. Methodol. Bull. 40, 30-36.

Eddens, K., Fagan, J.M., 2018. Comparing nascent approaches for gathering alter-tie data for egocentric studies. Soc. Networks 55, 130-141. https://doi.org/10.1016/j. socnet.2018.05.009.

Fischer, C.S., 1982. To Dwell Among Friends. Chicago University Press, Chicago.

Freeman, L.C., Romney, A.K., Freeman, S.C., 1987. Cognitive structure and informant accuracy. Am. Anthropol. 89, 310-325. https://doi.org/10.1525/ aa.1987.89.2.02a00020.

Goodreau, S.M., Kitts, J., Morris, M., 2009. Birds of a feather, or friend of a friend? Using exponential random graph models to investigate adolescent social networks. Demography 46, 103-125. https://doi.org/10.1353/dem.0.0045.

Green, H.D., Hoover, M.A., Wagner, G.J., Ryan, G.W., Ssegujja, E., 2014. Measuring agreement between egos and alters: understanding informant accuracy in personal network studies. Field methods 26, 126-140. https://doi.org/10.1177/ 1525822 X13492676.

Harling, G., Tanser, F., Mutevedzi, T., Bärnighausen, T., 2015. Assessing the validity of respondents' reports of their partners' ages in a rural South African population-based cohort. BMJ Open 5, 4-6. https://doi.org/10.1136/bmjopen-2014-005638.

Hogan, B., Janulis, P., Phillips, G.L., Melville, J., Mustanski, B., Contractor, N., Birkett, M., 2020. Assessing the stability of egocentric networks over time using the digital participant-aided sociogram tool network canvas. Netw. Sci. Camb. Univ. Press (Camb Univ Press) 8, 204-222. https://doi.org/10.1017/nws.2019.27.

Holbrook, A.L., Krosnick, J.A., Moore, D., Tourangeau, R., 2007. Response order effects in dichotomous categorical questions presented orally - the impact of question and respondent attributes. Public Opin. Q. 71, 325-348.

Jäger, A., 2005. Explaining the "accuracy"" of proxy-reports on attitudes towards immigrants in Germany. Two approaches compared". Metod. Zv. 2, 27-57.

Krackhardt, D., 1987. Cognitive social structures. Soc. Networks 9, 109-134. https://doi, org/10.1016/0378-8733(87)90009-8.

Krackhardt, D., Kilduff, M., 1999. Whether close or far: social distance effects on perceived balance in friendship networks. J. Pers. Soc. Psychol. 76, 770-782. https://doi.org/10.1037/0022-3514.76.5.770.

Krosnick, J.A., 1991. Response strategies for coping with the cognitive demands of attitude measures in surveys. Appl. Cogn. Psychol. 5, 213-236. https://doi.org/ 10.1002/acp. 2350050305.

Krosnick, J.A., 1999. Survey research. Annu. Rev. Psychol. 50, 537-567.

Krosnick, J.A., Presser, S., 2010. Questionnaire design. In: Wright, J.D., Marsden, P.V. (Eds.), Handbook of Survey Research. Emerald Group, West Yorkshire, England, pp. 263-314.

Krosnick, J.A., Holbrook, A.L., Berent, M.K., Carson, R.T., Hanemann, W.M., Kopp, R.J., Mitchell, R.C., Presser, S., Ruud, P.A., Smith, V.K., Moody, W.R., Green, M.C., 
Conaway, M., 2002. The impact of “no opinion”" response options on data quality Non-attitude reduction or an invitation to satisfice? Public Opin. Q. 66, 371-403.

Lee, F., Butts, C.T., 2020. On the validity of perceived social structure. J. Math. Psychol. 98, 102384 https://doi.org/10.1016/j.jmp.2020.102384.

Magaziner, J., Zimmerman, S.I., Gruber-Baldini, A.L., Hebel, J.R., Fox, K.M., 1997. Proxy reporting in five areas of functional status: comparison with self-reports and observations of performance. Am. J. Epidemiol. 146, 418-428. https://doi.org/ 10.1093/oxfordjournals.aje.a009295.

Marin, A., Hamilton, K., 2007. Simplifying the personal network name generator: alternatives to traditional multiple and single name generators. Field methods 19, 163-193.

Marineau, J.E., Labianca, G. (Joe), Brass, D.J., Borgatti, S.P., Vecchi, P., 2018. Individuals' power and their social network accuracy: a situated cognition perspective. Soc. Networks 54, 145-161. https://doi.org/10.1016/j. socnet.2018.01.006.

Marsden, P.V., 1990. Network data and measurement. Annu. Rev. Sociol. 16, 435-463. Marsden, P.V., 2011. Survey methods for network data. In: Scott, J., Carrington, P.J. (Eds.), The SAGE Handbook of Social Network Analysis. Sage, London, pp. 370-388.

Matza, L.S., Swensen, A.R., Flood, E.M., Secnik, K., Leidy, N.K., 2004. Assessment of health-related quality of life in children: a review of conceptual, methodological, and regulatory issues. Value Heal. 7, 79-92.

McPherson, M., Smith-Lovin, L., Cook, J.M., 2001. Birds of a feather: homophily in social networks. Annu. Rev. Sociol. 27, 415-444.

Menon, G., Bickart, B., Sudman, S., Blair, J., 1995. How well do you know your partner? Strategies for formulating proxy-reports and their effects on convergence to selfreports. J. Mark. Res. 32, 75-84.

Narayan, S., Krosnick, J.A., 1996. Education moderates some response effects in attitude measurement. Public Opin. Q. 60, 58-88. https://doi.org/10.1086/297739.

Pearcy, R., Waldron, D., O’Boyle, C., MacDonagh, R., 2008. Proxy assessment of quality of life in patients with prostate cancer: how accurate are partners and urologists? J. R. Soc. Med. 101, 133-138.

Perry, B.L., Pescosolido, B.A., Borgatti, S.P., 2018. Egocentric Network Analysis: Foundations, Methods, and Models. Cambridge University Press, Cambridge.

Peytchev, A., 2009. Survey breakoff. Public Opin. Q. 73, 74-97.

Pfenning, A., Mohler, P.P., Pfenning, U., 1991. Zur Reliabilität von egozentrierten Netzwerken in Massenumfragen. ZUMA Nachrichten 28, 92-108.

Roydhouse, J.K., Wilson, I.B., 2017. Systematic review of caregiver responses for patient health-related quality of life in adult cancer care. Qual. Life Res. 26, 1925-1954. https://doi.org/10.1007/s11136-017-1540-6.

Ryan, R.M., Deci, E.L., 2008. From ego depletion to vitality: theory and findings concerning the facilitation of energy. Available to the Self. Soc. Personal. Psychol. Compass 2/2, 702-717. https://doi.org/10.1111/j.1751-9004.2008.00098.x.

Schenk, M., 1995. Soziale Netzwerke und Massenmedien. Untersuchung zum Einfluß der persönlichen Kommunikation. J.C.B. Mohr, Tübingen.
Simpson, B., Markovsky, B., Steketee, M., 2011. Power and the perception of social networks. Soc. Networks 33, 166-171. https://doi.org/10.1016/j. socnet.2010.10.007.

Snijders, T.A.B., Bosker, R.J., 1999. Multilevel analysis. An Introduction to Basic and Advanced Multilevel Modeling. Sage, London.

Stark, T.H., 2018. Collecting social network data. In: Vannette, D.L., Krosnick, J.A. (Eds.), The Palgrave Handbook of Survey Research. Palgrave Macmillan, Cham, Switzerland, pp. 241-254. https://doi.org/10.1007/978-3-319-54395-6_31.

Stark, T.H., Flache, A., 2012. The double edge of common interest: ethnic segregation as an unintended byproduct of opinion homophily. Sociol. Educ. 85, 179-199. https:// doi.org/10.1177/0038040711427314.

Stark, T.H., Krosnick, J.A., 2017. GENSI: a new graphical tool to collect ego-centered network data. Soc. Networks 48, 36-45. https://doi.org/10.1016/j. socnet.2016.07.007.

Stocké, V., 2005. Einstellung zu Bildung. Zusammenstellung sozialwissenschaftlicher Items und Skalen. https://doi.org/10.6102/zis136.

Stocké, V., 2007. Explaining educational decision and effects of families' social class position: an empirical test of the breen-goldthorpe model of educational attainment. Eur. Sociol. Rev. 23, 505-519 https://doi.org/http://10.0.4.69/esr/jcm014.

Sudman, S., Bickart, B., Blair, J., Menon, G., 1994. The effect of participation level on reports of behavior and attitudes by proxy reporters. In: Schwarz, N., Sudman, S. (Eds.), Autobiographical Memory and the Validity of Retrospective Reports. Springer, New York, pp. 251-265.

Tetlock, P.E., 1983. Accountability and the perserverance of 1 st impressions. Soc. Psychol. Q. 46, 285-292.

Todorov, A., Kirchner, C., 2000. Bias in proxies' reports of disability: data from the national health interview survey on disability. Am. J. Public Health 90, 1248-1253.

Tourangeau, R., Rips, L.J., Rasinski, K., 2000. The Psychology of Survey Response. University Press, Cambridge.

Vehovar, V., Manfreda, K.L., Koren, G., Hlebec, V., 2008. Measuring ego-centered social networks on the web: questionnaire design issues. Soc. Networks 30, 213-222. https://doi.org/10.1016/j.socnet.2008.03.002.

White, K., Watkins, S.C., 2000. Accuracy, stability and reciprocity in informal conversational networks in Rural Kenya. Soc. Networks 22, 337-355.

Young, A.M., Rudolph, A.E., Su, A.E., King, L., Jent, S., Havens, J., 2016. Accuracy of name and age data provided about network members in a social network study of people who use drugs: implications for constructing sociometric networks. Ann. Epidemiol. 26, 802-809. https://doi.org/10.1016/j.annepidem.2016.09.010.

Yousefi-Nooraie, R., Marin, A., Hanneman, R., Pullenayegum, E., Lohfeld, L., Dobbins, M., 2019. The relationship between the position of name generator questions and responsiveness in multiple name generator surveys. Sociol. Methods Res. 48, 243-262. https://doi.org/10.1177/0049124117701484.

Zeggelink, E., 1995. Evolving friendship networks - an individual-oriented approach implementing similarity. Soc. Networks 17, 83-110. 